This is an authors' version of the chapter published in "Blockchain and Applications (Conference Proceedings)". The final authenticated version is available online at: https://doi.org/10.1007/978-3-030-23813-1.

\title{
The "tokenization" of the eParticipation in public governance: an opportunity to hack democracy.
}

\author{
Francisco Luis Benítez Martínez ${ }^{[0000-0002-2327-0746]}$ María Visitación Hurtado Torres ${ }^{[0000-}$ \\ ${ }^{0001-6345-6062]}$ Esteban Romero Frías ${ }^{[0000-0003-2205-3560]}$ \\ University of Granada, Spain \\ flbenitez@correo.ugr.es mhurtado@ugr.es erf@ugr.es
}

\begin{abstract}
Currently Distributed Ledger Technologies-DLTs, and especially the Blockchain technology, represent a great opportunity for public institutions to transform the channels of citizen participation and reinvigorate democratic processes. These technologies allow the simplification of processes and provide a strong security management of the data stored in its records, guaranteeing the transmission and public transparency of information. Therefore, the possibility of developing a new citizen governance model using this technology as a BaaS (Blockchain as a Service) platform emerges. G-Cloud solutions would facilitate a fast deployment in the cities and provide scalability to foster the creation of Smart Citizens within the philosophy of Open Government. The development of an eParticipation model that can configure a tokenizable system of the actions and processes that citizens exercise in democratic environments is an opportunity to guarantee greater participation and thus manage more effective local democratic spaces. Therefore, a Blockchain solution in eDemocracy platforms represents a great opportunity to claim a new pattern of management amongst the agents that participate in the public sphere.
\end{abstract}

Keywords: Governance, blockchain, eParticipation.

Reference: Benítez-Martínez, F. L., Hurtado Torres, M. V. \& Romero-Frías, E. (2019). "The "tokenization" of the eParticipation in public governance: an opportunity to hack democracy". In Prieto, J., Kumar Das, A., Ferretti, S., Pinto, A., \& Corchado, J. M. "Blockchain and Applications. International Congress" (pp. 110-117). Springer. 
This is an authors' version of the chapter published in "Blockchain and Applications (Conference Proceedings)". The final authenticated version is available online at: https://doi.org/10.1007/978-3-030-23813-1.

\section{Introduction}

Since 2009 when Satoshi Nakamoto [1] described the procedures to use Blockchain technology as a cryptographic management system in a single and distributed registry, he opened the way to transform not only the economy but a large part of our social and political system.

Thanks to this, the development of applications in the Fintech sector has continued to be deployed in the Internet with a set of dApps (mobile applications using blockchain technology that can be public or permissioned) that allow its multi-screen integration in a distributed manner. In parallel, there are new types of DLTs that progress in the development of new formats and possibilities due to more advanced node topologies such as Hedera Hashgraph [2] or Tangle, from the IOTA Foundation [3].

Developing a DLT is an opportunity to manage complex processes that simplify governance practices. They can guarantee the structure and security of the data. They are an opportunity for the management of transparency and transactions that take place in public administrations. Another important feature is the capacity of its temporary sealing and the inalterability of the data it contains. It allows the configuration of effective management systems [4] to achieve better accountability, through standard and scalable eGovernment models at all territorial levels of the administration.

For all these reasons, we currently have a technology that can develop a new democratic management framework to empower citizens and establish new participation relationships that go beyond voting or administrative management.

The digital transformation of the administration in the service of citizen empowerment is a governmental obligation and a civic demand that is possible and necessary, not only because of the disruption that the DLTs suppose, but also the Cloud solutions and the possibility of managing the tools on platforms. There are methodologies for this, such as the one proposed by Huang and Karduck [5] and we have the opportunity to experiment with new solutions for democratic processes that allow new management systems for public governance.

Therefore, Blockchain technology becomes a unique opportunity to manage new governance processes in the environments of public institutions for the improvement of their efficiency and effectiveness [6] especially if it is used as a platform [7].

The objective of this paper is to propose a new model of eParticipation system that integrates the possibilities that DLT systems [8] have, in relation to public governance processes and the proposal of new methods to develop new tools that allow citizen empowerment To this end, we will describe a "tokenizable" participation system that helps local administrations to improve participatory projects using electronic voting tools based on blockchain that allows an improvement in citizen participation when it comes to collecting their participation in the management of the regular administration of cities.

The use of a G-Cloud system will be proposed to allow its optimal deployment with a high capacity of scalability and reproducibility in any environment, following a proven methodology for it [9]. 
This is an authors' version of the chapter published in "Blockchain and Applications (Conference Proceedings)". The final authenticated version is available online at: https://doi.org/10.1007/978-3-030-23813-1.

This paper proposes the application of new processes and procedures with the use of blockchain as a platform within a G-Cloud based framework, providing citizenship with engagement in local eParticipation projects. The structure of the paper is as follows. The next section presents a literature review of successful platforms used in the study in order to present the proposed model. The third section presents the model proposed from the perspective of the lack of new ways of governance in the Smart City context and the concrete idea of a tokenizable eParticipation model. The paper ends with a conclusion about the opportunity of a new eParticipation model using blockchain as a platform to engage citizen participation and finally offers directions for future work.

\section{Technological Background and related works}

Currently there is a series of platforms and tools developed that can be the technological basis for the development of different governance projects in institutions and companies. Below we describe the most relevant:

1. Bitnation [10] is a decentralized platform for Governance 2.0. It is a political experiment from a voluntary, transnational and borderless perspective, which explores new democratic and social processes using the Blockchain technology. This platform was deployed in July 2014. In it, the first marriage using this technology was managed, alien to traditional institutions. Also, new systems of personal identification outside the control of the state and legal systems with digital notaries within it are experimented with. For the development of these social innovations, the Pangea project is used, which is a decentralized market for legal services, where users can experience new services of citizen governance. All their Smart Contracts are implemented in Ethereum.

2. Follow My Vote [11] is designed as a platform to guarantee electronic voting with high security, allowing a high degree of transparency in the democratic processes in which it is used. It can also be developed without compromising the privacy of the voters who use it, guaranteeing the traceability of their vote throughout the process until the closing of the voting and the final results. It is developed in open code, so anyone can audit the code used. It has been deployed both in UN votes and at a less rigid level, such as the television show "The Voice" in its North American version.

3. BoardRoom [12] is a governance management system that integrates both a dashboard and a dApp, which can be used both individually and by companies to manage their Smart Contracts, equally in public and permissioned blockchains developed in Ethereum. It serves both to issue tokens in crowdfunding projects, proxy voting systems for shareholders in Committees or Shareholders' Meetings and the development of payment systems through tokens in industrial consortiums. 
This is an authors' version of the chapter published in "Blockchain and Applications (Conference Proceedings)". The final authenticated version is available online at: https://doi.org/10.1007/978-3-030-23813-1.

4. Consul [13] is the most developed citizen participation tool to date for open government projects in municipal environments. It is developed in free software (its code is complete in GitHub) and has established the DECIDE platform to manage the eParticipation systems that it integrates. It is in use in 33 countries, and is used by 100 institutions such as the municipalities of Madrid (Spain), Paris (France) and Buenos Aires (Argentina). It has a main advantage, in that it has allowed the development of a scalable and highly tested system in several institutions around the world, but despite a high degree of reliability it can not guarantee the level of privacy and transparency that would allow it to be used as a standard.

5. TIVI [14] is the platform developed by Smartic and Cybernetica for verifiable voting using biometric factors from the voting point. Similarly to Consul it does not use any type of DLT and uses encryption systems to secure the votes. They have developed voter authentication systems in Argentina and Armenia in 2017 and their first inclusion in systems of choice with Blockchain technology was in 2016 for the Primary elections of the Republican Party of Utah in the United States. Their actions are more oriented to electoral automatization processes than to the development of eParticipation systems.

\section{New approach for a "Tokenizable" e-Participation model}

\subsection{The Governance deficit}

Currently the concept of governance is a word devoid of meaning. Society does not perceive a risk management system and the uncertainty generated by globalization. Within a system where profits are minimized and losses are maximized, it is critical to redefine what good governance consists of.

It is necessary to establish a new governance system that develops a participatory system of governance, taking advantage of the technological opportunities that we have today. And this concept allows civic and technological empowerment to citizenship.

Governance is, in short, the construction of a proactive citizen governance, that is, a citizen with the capacity and possibility of taking an active part in the daily government, thanks to the development of a new democratic model through the use of the tools that the eDemocracy provides.

\subsection{The role of Open Smart Cities and the "smart citizens": opening local governments.}

Our approach is part of the open government systems that have a series of digital tools for the development of eDemocracy solutions in the ambit of public participation. Therefore, this approach requires reconsidering the relationship between technology, people and cities from a perspective centered on citizenship and with its complex and contradictory vision of the urban concept [15]. 
This is an authors' version of the chapter published in "Blockchain and Applications (Conference Proceedings)". The final authenticated version is available online at: https://doi.org/10.1007/978-3-030-23813-1.

The philosophy of the Smart City, whose development has been possible from the point of view of technology and Big Data, has led to its decision-making being replaced by a technocratic decision system based on the dictatorship of isolated data, and not from the point of view of the development of consensual public policies. Therefore, in the same way that the Open Government concept has been developed to establish new participation and accountability systems, it is necessary to establish a new concept: the Open Smart City.

Open Smart City is a philosophy that implies empowering citizens, that is, putting cities at the service of their citizens, empowering, in turn, public officials to develop the policies that are defined and agreed upon by the parties. It is the paradigm of governability (the form of governing), which not only governance (the processes and protocols that allow governing), which requires a more complex and permanent effort.

This vision implies giving space to new dynamics that come from society as citizen science, digital activism, "artivism", the "maker" movement, the policy and social labs [16], the co-creation and self-development communities, digital citizen movements, social hackers, citizen innovation laboratories [17], etc.

The idea presented aims to "open" the data and the processes that generate cities to make them available to society, becoming a common practice in the service of people. It provides an opportunity to create more democratic co-decision spaces in the political arena, creating the figure of Open Smart Citizens, i.e. citizens who operate in the spaces described above and that offer a new opportunity to generate new public policies and new democratic spaces.

Data mining and big data allow the development of new tools and techniques for the management of the city and its social diversity, from the point of view of social innovation.

Our proposal aims to support the growing demand of citizens to participate actively in the decisions that affect cities and the evolution of data mining techniques and their visualization tools. These allow the availability of information in real time, giving the people the possibility of having immediate data to manage a personal responsibility in the creation and design of services that affect them directly [18]. These processes of co-creation and co-participation are in the fundamental principles of open government and are what allow the deployment of the elements that form the Open Smart Cities format.

\subsection{A "tokenizable" eParticipation model}

The model we propose (Fig.1) is based on a public G-Cloud system that allows the integration of a blockchain platform as a service (BaaS). This guarantees the functional requirement of scalability necessary in any governmental and / or territorial environment. In our system, the voter would access the platform through a permissioned dApp that would connect with the interface and the voter database to proceed with its recognition and identify him / her in the process. 
This is an authors' version of the chapter published in "Blockchain and Applications (Conference Proceedings)". The final authenticated version is available online at: https://doi.org/10.1007/978-3-030-23813-1.

Then the System Administrator would activate the Smart Contract in the front end that would allow the citizen to participate in the process opened by the agency to register their participation and the meaning of their vote. In the validation node, the encryption of the data is performed with a one-way hash function SHA-256 so that the result of the voting cannot be reversed. Once the vote is encrypted, the block will be added to the blockchain within the BaaS architecture used, and in turn the block generates the "token" that will grant the voter a series of advantages and / or rights of use in the city.

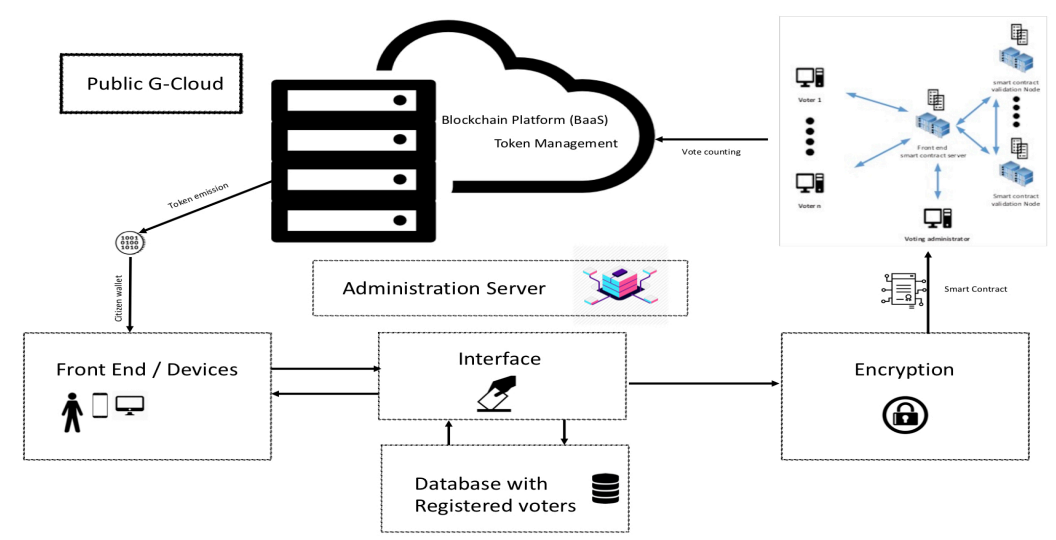

Fig. 1. Simplified representation of a Blockchain e-Voting participation

For the development of this proposal we have been taken into account previous studies of electronic voting that are being done in parallel by blockchain to solve different technical issues for its progressive implementation. Specifically:

a. Those that differentiate the use of blockchain for the development of voting systems using crypto currencies [19], such as those that use the Zcash protocol [20],

b. Those that rely on the deployment of Smart Contracts [21] with the limitations of options and number of voters that this entails, as is the case of BoardRoom,

c. And those that manage the blockchain as a "ballot box" as FollowMyVote and TIVI do [22].

Also there have been studies that have developed electronic voting systems that establish taxonomies and requirements $[23,24]$, the conceptualization of how to use blockchain to develop voting systems [25] or how to develop voting systems that allow more control of the process and its decisions to voters [26].

Taking into account that for the development of fintech projects for cryptocurrencies there is a large repository of projects and academic articles on their implementa- 
This is an authors' version of the chapter published in "Blockchain and Applications (Conference Proceedings)". The final authenticated version is available online at: https://doi.org/10.1007/978-3-030-23813-1.

tion, it is an opportunity to take advantage of all that experience to use "tokens" in a practical way in eDemocracy environments.

The idea proposed is to use "tokens" to reward the participation and comanagement of projects of public utility.

The procedures to which it can be applied are varied, from accumulating them to exchange them for discounts in taxes or municipal fees for the participation in voting, the possibility of exchanging them to obtain cultural or sports advantages (tickets, subscriptions, discounts) or for the demonstration of the development of civic values through participation in the deployment of public policies.

With this proposal, a whole new field of citizen management and work is opened, which will help to increase participation ratios in the public processes and votes that are proposed, as we have been working in the MediaLab of the University of Grana$\mathrm{da}$, as well as in projects with the City of Granada on social innovation and comanagement of public policies. We believe that it is a possibility to manage new developments of social innovation based on the deployment of a platform that is conceived as such. This will increase citizen participation and especially of the young population, by making use of dApps in any digital format.

\section{Conclusions and Future Work}

Currently it is necessary to experiment with new methods that allow a greater commitment of citizens to their local institutions and the blockchain technology allows it, thanks to one of its representative elements: the token.

In this work, a model based on blockchain has been proposed for the management of local governance, as a more efficient and inclusive way that opens new paths for the local management of territories. Its deployment follows a BaaS approach, which ensures scalability in a G-Cloud solution environment. This approach enables the development of eDemocratic environments that allow better management and more citizen engagement.

The proposal is based on the use of "Tokens" to increase and reward the participation of the citizen and to promote the co-management of public utility projects.

It is aimed at finding new forms of commitment that can strengthen local democracies, as the technological deployment in the past decade has not been accompanied by greater participation or by a greater resilience of our democracies to the spaces of uncertainty generated by our society.

In section 2, different solutions have been presented that corroborate the technological possibility of carrying out new steps in this direction, in order to address the existing deficit of citizen governance.

The blockchain technology applied to governance processes is yet to be developed. Therefore, there is an opportunity to experiment with new tools and new eParticipation formats to help consolidate and reform our democratic systems and to make them stronger and more participatory. 
This is an authors' version of the chapter published in "Blockchain and Applications (Conference Proceedings)". The final authenticated version is available online at: https://doi.org/10.1007/978-3-030-23813-1.

Currently, we are working on the development of a participation system based on this proposal for the City of Granada. We have the participation of the MediaLab of the University of Granada which is part of the project of the Integrated Sustainable Urban Development that must develop a participatory project as an urban innovative action.

We plan to use the UTAUT model (Unified Theory of Acceptance and Use of Technology) [27] to understand how this new eParticipation model will be valued among the citizens who will use it.

In parallel, we are the coordinators of the Working Group of the Association of Spanish Scientific and Technological Parks (APTE) that will launch the first permissioned blockchain network in the service of public research centers and R \& D companies that are established in its network (53 parks), in order to develop new management processes, new dApps and disruptive solutions in different markets.

In both projects the "participatory tokens" are a strategic element for the results that are expected. These results will be the indicators of the degree of success of the model presented in this work, in the 2020 horizon.

\section{References}

1. Nakamoto, S. Bitcoin: A peer-to-peer electronic cash system. (2008), https://bitcoin.org/files/bitcoin-paper/bitcoin_es.pdf, last accessed 2019/2/7

2. HEDERA Hashgraph Plattform, https://www.hedera.com/platform, last accessed 2019/2/7

3. IOTA Foundation Tangle protocol, https:/www.iota.org/research/meet-thetangle, last accessed 2019/2/7

4. Alexopoulos, C., Charalabidis, Y., Androutsopoulou, A.: Benefits and Obstacles of Blockchain Applications in e-Government. In: Proceedings of the $52^{\text {nd }}$ Hawaii International Conference on System Sciences, pp. 3377-3386. (2019).

5. Huang, J., Karduck, A.: A Methodology for Digital Government Transformation. In: Journal of Economics, Business and Management, vol. 5, no. 6, pp. 246-254 (2017).

6. Morabito, V.: Blockchain governance. In: Business innovation through Blockchain: The $\mathrm{B}^{3}$ Perspective, pp. 41-59. Springer International Publishing, (2017).

7. Glaser, F. et al.: Blockchain as a Platform. In: Business Transformation through Blockchain. Vol I. Treiblmaier, H. and Beck, R. (eds.). Palgrave MacMillan. Pp. $121-143$ (2019).

8. Rauchs, M. et al.: Distributed Ledger Technology Systems. A conceptual framework. Cambridge Centre for Alternative Finance. (2018).

9. IIIT Hyderabad.: Cloud Computing for e-Governance. White Paper. (2010), http://search.iiit.ac.in/uploads/CloudComputingForEGovernance.pdf, last accessed 2019/2/8.

10. Bitnation Governance 2.0 Platform: https://tse.bitnation.co/, last accessed 2019/2/9

11. Follow My Vote Platform: https://followmyvote.com/, last accessed 2019/2/9

12. BoardRoom Platform: http://boardroom.to/\#About, last accessed 2019/2/9

13. CONSUL Project: http://consulproject.org/en/index.html, last accessed 2019/2/9 
This is an authors' version of the chapter published in "Blockchain and Applications (Conference Proceedings)". The final authenticated version is available online at: https://doi.org/10.1007/978-3-030-23813-1.

14. TIVI Online Voting System: https://tivi.io/, last accessed 2019/2/9

15. Fernández, M.: Descifrar las Smart Cities: Qué queremos decir cuando hablamos de Smart Cities, pp. 183-185. Caligrama Editorial, Madrid (2016).

16. Romero-Frías, E., Arroyo-Machado, W. (2018). Policy Labs in Europe: Political Innovation, Structure and Content Analysis on Twitter. In: El profesional de la información, 27, (6), pp. 1181-1192 (2018).

17. LabIn Granada. Social Innovation Lab. https://labingranada.org/, last accessed 2019/2/10.

18. Garriga-Portolá, M.,López Ventura, J.: The role of Open Government in Smart Cities. In: Open GovernmentPublic Administration and Information Technology 4. Springer Science + Business Media, New York. (2014).

19. Zhao, Z., Chan, T.H.H.: How to vote privately using bitcoin. In: International Conference on Information and Communications Security. Pp. 82-96. Springer (2015).

20. Hopwood, D. et al.: Zcash protocol specification. Tech. rep., 2016-1.10. Zerocoin Electric Coin Company (2016).

21. McCorry, P., Shahandashti, S.F., Hao, F.: A smart contract for boardroom voting with maximum voter privacy. IACR Cryptology ePrint Archive 2017, 110 (2017).

22. Yu, B. et al.: Platform-independent Secure Blockchain-Based Voting System. In: Chen L., Manulis M., Schneider S. (eds) Information Security. ISC. Lecture Notes in Computer Science, vol 11060. Springer, Cham, pp. 369-386 (2018).

23. Annae, R., Freeland, R. and Theodoropoulos, G.: E-voting requirements and implementation. In: The $9^{\text {th }}$ IEEE CEC/EEE 2007. IEEE, 2007, pp. 382-392 (2007).

24. Wang, K.H. et al.: A review of contemporary e-voting: Requirements, technology, systems. In: Data Science and Pattern Recognition, vol. 1, no. 1, pp - 31-47 (2017).

25. Ayed, A.b.: A conceptual secure blockchain-based electronic voting system. In: International Journal of Network Security \& Its Applications, vol. 9, no. 3 (2017)

26. Hardwick, F.S., Gioulis, A., Akram, R.N.: E-Voting with Blockchain: An e-Voting Protocol with Decentralisation and Voter Privacy. ISG-SCC, Royal Holloway, University of London, UK. (2018).

27. Naranjo-Zolotov, M. et al.: Examining social capital and individual motivators to explain the adoption of online participation. In: Future Generation Computer Systems 92, pp. 302311. Elsevier B.V. (2018). 